\title{
Force control of an underactuated prosthetic hand based on sliding mode with exponential reaching law
}

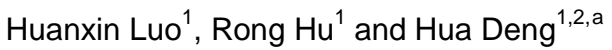 \\ ${ }^{1}$ School of Mechanical and Electrical Engineering, Central South University, Changsha 410083, China \\ ${ }^{2}$ State Key Laboratory of High Performance Complex Manufacturing, Changsha 410083, China
}

\begin{abstract}
The design of a sliding mode force control system for an underactuated prosthetic hand system are addressed in this study. First, a mathematical model of underactuated prosthetic hand is developed and state equation of DC motors is discussed for force control. Next, sliding mode force control based on exponential reaching law was discussed to increase the control response. Finally, the proposed control strategy for underactuated prosthetic hand is simulated to grasp soft, medium or firm objects. The results verify the effectiveness of the force control method based on sliding mode with exponential reaching law.
\end{abstract}

Keywords: force control; underactuated prosthetic hand; sliding mode control.

\section{Introduction}

In order to improve the life quality of amputees, providing approximate manipulation ability of a human hand to that of a prosthetic hand is considered by many researchers [1]. Researchers have begun to identify methods of making prosthetic hands both functional and cosmetically appealing.

The commercial prosthetic hand which has one DOF grasp an object using proportional control method. The underactuated prosthetic hand is useful for adaptive grasping, this underactuated mechanism has been developed with tendon-driven mechanisms, using both actuators and passive springs [2].The underactuated prosthetic hand is useful to grasp different objects. However, it is difficult to the force control because little force will drop the grasped objects and great force will deform it [3],[4].

Nonlinear model predictive control (NMPC) is used to provide feedback control to the prosthetic device. It utilizes a neural network to model the dynamics of each finger. Using this model, it is possible to predict the amount of force exerted by the prosthetic hand [5] .An adaptive fuzzy proportional-integral-derivative (PID) force control strategy is presented for a prosthetic hand [6]. Force-derivative feedback has also been used in conjunction with a fuzzy logic controller to reduce unwanted oscillations that occur during force control of an underactuated finger [7]. To produce a more natural feeling controller, Normal force-derivative feedback to hand is used to prostheses. The normal force derivative is obtained by numerically differentiating the measured normal force [8]. [911] presents a controller for a multi-fingered underactuated prosthetic hand. The pre-shaping of the hand using PID position control algorithms. The desired force closure value is calculated according to the pre-shape measured tendons tension and the grasping force.

${ }^{a}$ Corresponding author : hdeng@cus.edu.cn

(C) 2016. The authors - Published by Atlantis Press 
For prosthetic hands, one of important work is to design a proper control strategy [12]. Sliding mode controllers based on constant reaching law are implemented for a four-link prosthetic hand [13, 14], the control method with constant reaching law is fast to response because the rigidity of this prosthetic hand is large. However, the underactuated prosthetic hand has complicated mechanism, flexible joint and cable transmission, this generally introduce a large friction force that degrades the control response [15].So, sliding mode with exponential reaching law which has quick response to system is fit for underactuated prosthetic hand [16].

In this paper, a sliding mode force control based on exponential reaching law is presented. The model of underactuated prosthetic hand system is developed in Section II.The system consists of DC micro motor and an underactuated prosthetic hand. Lagrange formulation applied to find the dynamic model of the underactuated prosthetic hand. State equation of DC motors is discussed for coming sliding mode control method. Sliding mode force control based on reaching law was discussed in Section III. The sliding mode control introduced to the prosthetic hand system retained the robustness of the control system. The computer simulation results shown in Section IV, it verifes the validity of the sliding mode control method based on reaching law.

\section{Underactuated prosthetic hand system}

The prosthetic hand is composed of a DC micro motor and an underactuated prosthetic hand. The hand has three degrees of freedom. The fingers are derived by DC micro motor through a rod. The model scheme of the underactuated prosthetic hand system is shown in Fig. 1, where $u$ is the input voltage of DC motor; $T_{L}$ is the load torque on the motor; $F_{2}$ denotes the force exerted by the environment; and $x$ denotes the distance between the environment and tips.

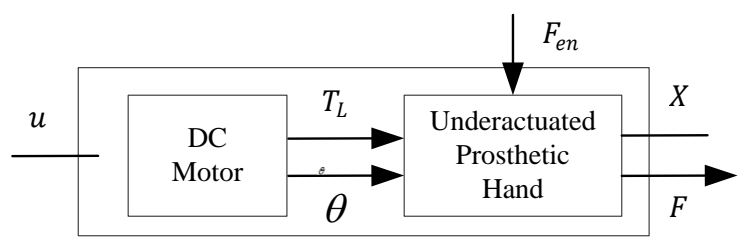

Figure 1. Model scheme of an underactueated prosthetic hand system.

\subsection{The mathematical model of underactuated prosthetic hand}

As shown in Fig.2, the finger based on underactuated mechanism has three joints. Only the base joint $\mathrm{i}$ s presented with the acutuator while the others are driven, which makes the smaller finger harder to co ntrol. Under the occation that the object is close to the center of the palm, the proximal phalanx touche $\mathrm{s}$ and is hindered, subsequently, the middle and distal phalanx continue their movements until the obje ct is fully surrounded. The grasping control system has highly adaptivity to adapt objects with almost all shapes enhancing the capability [17].

The finger has three DOF actuated by a DC motor, which flexes the fingers by pulling cables extending to the fingertips. As shown in Fig. 2, a bidimensional mathematical modle has been developed in order to evaluate the finger dynamic behavior during the reaching phase to the objects.

The finger dynamic model can be written starting from the Lagrangian formulation. The equation of dynamics of an underactuated prosthetic finger in Cartesian coordinates can be obtained in the form:

$$
M_{x}(q) \ddot{x}+C_{x}(\dot{q}, q) \dot{x}+G_{x}(q)+F_{x}=F_{\tau}
$$

Where $M_{x}(q)$ is a 33 positive definite symmetric matric, $C_{x}(, q)$ is a 31 centrifugation and Coriolis vector and $G_{x}(q)$ is a 31 elastic vector in Cartesian coordinates. $F_{\tau}$ is the driving torque matrix of joints, $F_{X}$ is external force. 


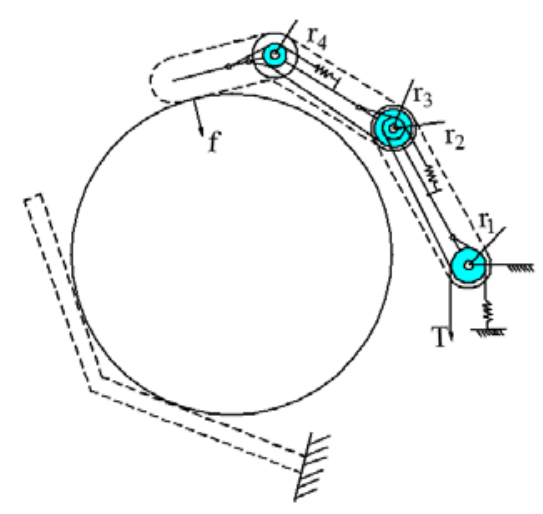

Figure 2. Finger structure.

The contact force between the finger and the environment can be simplified as a constant stiffness spring model[18,19], that is

$$
f=K_{e v} x
$$

Where $K_{e v}$ is the environment stiffness, $x$ is the vertical displacement of the prosthetic finger at the contact point.

The environment stiffness is the combination stiffness between grasped object and silicone glove of finger. The environment stiffness can be calculated as

$$
K_{e v}=\frac{K_{x} K_{w}}{K_{x}+K_{w}}
$$

Where $K_{x}$ is stiffness of silicone glove of finger, $K_{w}$ is stiffness of grasped object.

When the underactuated prosthetic hand contact with the environment, we assume the contact point is the equilibrium position of the prosthetic hand system, so the displacement $x$ can be approximated by

$$
x=f\left(\theta_{1}, \theta_{20}, \theta_{30}\right)
$$

Where ${ }_{20},{ }_{30}$ is the second and third rotational joint angle when the finger contact with the environment, ${ }_{1}$ is the motor rotation angle.

\subsection{The state equation of DC motor}

The schematic diagram of DC motor circuit can be shown in Fig. 3,the voltage of motor is comprise of resistance voltage, inductance voltage and back EMF voltage [20] .

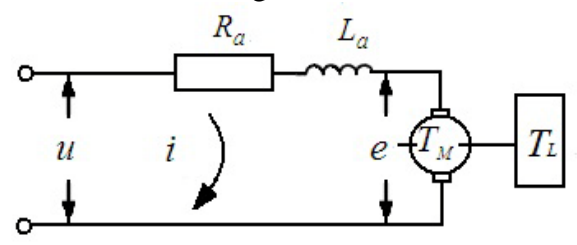

Figure 3. Schematic diagram of DC motor circuit.

Inductance voltage equation of DC motor is 


$$
L_{a} \frac{d i}{d t}+R_{a} i_{a}+K_{e} \omega=u
$$

Where $L_{a}$ is rotor inductance, $i$ is current of armature circuit, $R_{a}$ is terminal resistance of the motor, $u$ is input voltage to motor, $K_{e}$ is Back-EMF constant, is the rotor angular velocity.

Torque balance equation of DC motor is

$$
J \frac{d \omega}{d t}+B_{m} \omega=K_{t} i-T_{L}
$$

Where $J$ is inertia of motor, $B_{m}$ is viscosity coefficient of motor, $K_{t}$ is tongue constant, $T_{L}$ is load torque on motor.

The dynamic model of the prosthetic hand system which is composed of the motor model and the prosthetic finger is built by Eq. 1, Eq. 2, Eq. 4, Eq. 5 and Eq. 6.

Assigning state variables $x_{1}, x_{2}$ and $x_{3}$ corresponding to, $i$, respectively, yield the following form of the system model:

$$
\begin{gathered}
\dot{x}_{1}=-\frac{B}{J} x_{1}+\frac{K_{t}}{J} x_{2}-\frac{K_{e s}}{J} x_{3} L(\theta) \\
\dot{x}_{2}=-\frac{K_{e}}{L_{a}} x_{1}-\frac{R_{a}}{L_{a}} x_{2}-\frac{1}{L_{a}} u \\
\dot{x}_{3}=x_{1}
\end{gathered}
$$

Where $L(\theta)$ is the arm of external force. When the finger contact grasped object, $L(\theta)$ is constant which related to three joint angles.

The state space equation of the system is

$$
\dot{x}=A x+B u
$$

Where $A=\left[\begin{array}{crc}-\frac{B_{m}}{J} & \frac{K_{t}}{J} & -\frac{K_{e s}}{J} L(\theta) \\ -\frac{K_{e}}{L_{a}} & -\frac{R_{a}}{L_{a}} & 0 \\ 0 & 0 & 0\end{array}\right], B=\left[\begin{array}{c}0 \\ \frac{1}{L_{a}} \\ 0\end{array}\right]$

\section{Sliding mode force control based on reaching law}

A sliding mode force control is designed as shown in Fig.4. The single input is desired force $F_{d}$, the desired displacement $x_{d}$, can be calculated as

$$
x_{d}=\left(F_{d}-f\right) K_{N}
$$

Where $K_{N}$ is stiffness gain, $f$ is the contact force with environment. 


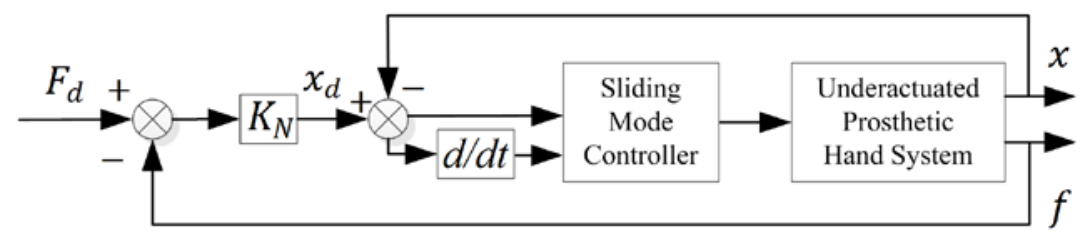

Figure 4. Schematic structure of sliding mode force control system.

In the force feedback loop, sliding mode force controller is designed to improve robustness, or reduce sensitivity of deviations in system parameters and external disturbances. A sliding mode controller is described by the following model

$$
s=\dot{e}+c e
$$

Where $c>0$.

The position error is

$$
e=x_{d}-x, \quad \dot{e}=\dot{x}_{d}-\dot{x}
$$

Integrating (12) and (13), yields

$$
\begin{aligned}
& \dot{s}=\ddot{e}+c \dot{e}=\left(\ddot{x}_{d}-\ddot{x}\right)+c\left(\dot{x}_{d}-\dot{x}\right) \\
& =\left(\ddot{x}_{d}-f\left(\ddot{x}_{3}, \theta_{20}, \theta_{30}\right)\right)+c\left(\dot{x}_{d}-f\left(\dot{x}_{3}, \theta_{20}, \theta_{30}\right)\right)
\end{aligned}
$$

Adapt exponential reaching law

$$
\dot{s}=-\varepsilon \operatorname{sgn} s-k s \quad \varepsilon>0, k>0
$$

Integrating (14) and (15), yields

$$
\left(\ddot{x}_{d}+f(x, t)-b_{2} u\right)+c\left(\dot{x}_{d}-f\left(\dot{x}_{3}, \theta_{20}, \theta_{30}\right)\right)=-\varepsilon \operatorname{sgn} s-k s
$$

In this controller, $u$ can be rewritten as

$$
u=\frac{1}{b_{2}}\left(\varepsilon \operatorname{sgn} s+k s+c\left(\dot{x}_{d}-f\left(\dot{x}_{3}, \theta_{20}, \theta_{30}\right)+\ddot{x}_{d}+f(x, t)\right)\right.
$$

Now, the sliding mode controller used to track a desired force, it has great robust in the sense that it can still guarantee excellent tracking performance in spite of model inaccuracies and the presence of unknown, possibly nonlinear and time-variant disturbances acting on the system.

\section{Simulations}

To verify the proposed approach to grasp objects with different stiffness, the adaptation grasping of the underactuated prosthetic finger has been simulated in MATLAB. We assumed ten centimeters cylinder that can be contacted by the underactuated prosthetic finger. The cylinder is simulated under the stiffness $K_{e v}$ of environment. We select three kinds of desired grasp force corresponding to soft, medium or firm grasped objects. The cylinder is fixed in work space. Three value of environment stiffness $K_{e v}$ have been settled to simulate soft, medium stiffness and hard object.

Case 1: The adaptation grasping soft objects simulation with the desired force from $2 N$ to $3 N$. The grasp force, the force error and control input under $K_{e v}=0.5 \mathrm{~N} / \mathrm{mm}$ stiffness are shown in Fig. 5. 

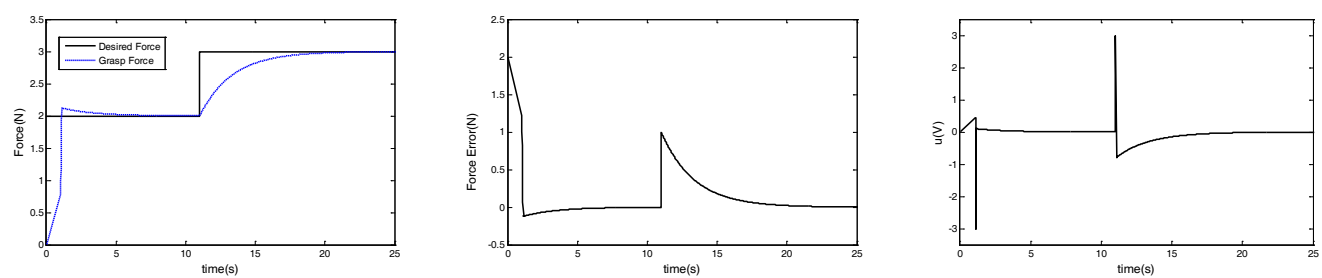

Figure 5. Force, force error and control input results from the simulation with case 1.

Case 2: The adaptation grasping medium objects simulation with the desired force from $3 N$ to $5 N$. The grasp force, the force error and control input under $K_{e v}=2 N / m m$ stiffness are shown in Fig. 6 .
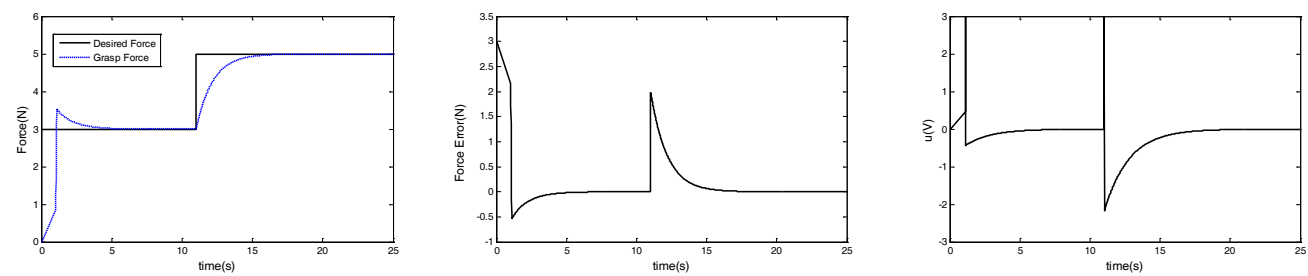

Figure 6. Force, force error and control input results from the simulation with case 2.

Case 3: The adaptation grasping hard objects simulation with the desired force from $5 \mathrm{~N}$ to $10 \mathrm{~N}$. The grasp force, the force error and control input under $K_{e v}=5 \mathrm{~N} / \mathrm{mm}$ stiffness are shown in Fig. 7.
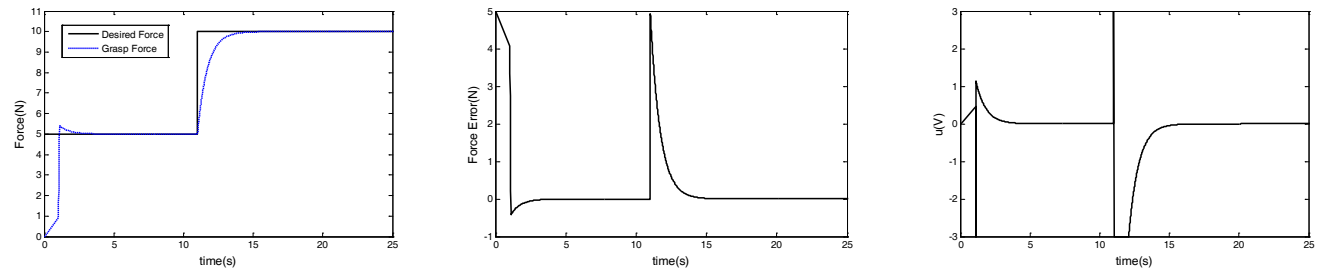

Figure 7. Force, force error and control input results from the simulation with case 3

The simulation results with different environment stiffness (Fig. 5 Fig. 7) show that proposed sliding mode force control method has a good control performance for the underactuated prosthetic system. When the proximal phalanx contacts with the object, the force between the finger and environment reaches to the desired force. Contrastive analysis of Fig. 5, Fig. 6and Fig. 7 indicate that the amplitude of force increase with the environment stiffness improvement. The gradient of force increment related to environment stiffness. Contrastive analysis of Fig. 5, Fig. 6 and Fig. 7 indicate that the force error is close to zero. The error is big at the point of desired force increment because we assume grasped object as a virtual spring which has large deformation.

\section{Conclusion}

A sliding mode force control method is presented to control underactuated prosthetic hand. The model of the prosthetic hand system is established for the control system. Slide mode control based on exponential reaching law applies to grasp force control. Three value of environment stiffness are simulated by the presented control method, the simulation results demonstrate the effectiveness of the sliding mode control method.

\section{Acknowledgment}

This research was supported by the National Basic Research Program of China (973 project: 2011CB013302). 


\section{References}

1. Arslan Y Z, Hacioglu Y, Yagiz N. Prosthetic Hand Finger Control Using Fuzzy Sliding Modes [J]. Journal of Intelligent \& Robotic Systems, 52(1):121-138(2008)

2. Ozawa R, Kobayashi H, Hashirii K. Analysis, Classification, and Design of Tendon-Driven Mechanisms [J]. IEEE Transactions on Robotics, 30(2):396-410(2014).

3. M.C.Carrozza, G.Cappiello,S.Micera,B.B.Edin,and C.Cipriani,”Design of a cybernetic hand for perception and action,”Biological Cybernetics, 95,629-644(2006).

4. A.Muzumdar, Powered Upper Limb Prostheses. Berlin, Germany: Springer, (2004).

5. Pasluosta C F, Tims H, Chiu A W L, et al. Slippage Sensory Feedback and Nonlinear Force Control System for a Low-Cost Prosthetic Hand[J]. American Journal of Biomedical Sciences, 1(1):295-302(2009).

6. Zhu G K, Duan X G, Deng H. Adaptive Fuzzy PID Force Control for a Prosthetic Hand[J]. Applied Mechanics \& Materials, 433-435:93-101(2013).

7. Birglen L, Gosselin C M, Birglen L, et al. Fuzzy Enhanced Control of an Underactuated Finger Using Tactile and Position Sensors[J]. 2005:2320-2325.

8. Engeberg E D, Meek S. Improved grasp force sensitivity for prosthetic hands through forcederivative feedback [J]. IEEE transactions on bio-medical engineering, 55:817-21(2008).

9. B. Massa,S. Roccella, M Carrozza, and P. Dario,’Design and development of an underactuated prosthetic hand,"in Proc,IEEE Int Conf, Robot. Autom, vol.4, pp.3374-3379(2002).

10. L. Zollo, S. Roccella, E. Gualielmelli, M.Carrozza, and P. Dario,”Biomechatronic design and control of an anthropomorphic artificial hand for prosthetic and robotic applications,”IEEE/ASME Transactions on Mechatronics, vol, 12, pp.418-429, (2007).

11. J. Zhao, Z. Xie, L. Jiang, H. Cai, H. Liu and G. Hirzinger, "A five-fingered underactuated prosthetic hand control scheme", Proc. IEEE International Conference on Biomedical Robotics and Biomechatronics, pp:995-1000, (2006).

12. N. Dechev, W. L. Leghorn, S. Neumann, "Multiple finger, passive adaptive grasp prosthetic hand," Mechanism and Machine Theory 36(2001):1157-1173.

13. Engeberg E D, Meek S G. Adaptive sliding mode control of grasped object slip for prosthetic hands.[C] Proceedings of the. IEEE/RSJ International Conference on Intelligent Robots and Systems. 2011:4174-4179.

14. Engerber, ED., Meek, S.G., Minor, M.A.: Hybrid force-velocity sliding mode control of a prosthetic hand. IEEE Trans. Biomed. Eng.55 (5), 1572-1581(2008).

15. Kaneko M, Yamashita T, Tanie K. Basic considerations on transmission characteristics for tendon drive robots[C] International Conference on Advanced Robotics, 1991:827-832 vol.1.

16. Fallaha C J, Saad M, Kanaan H Y, et al. Sliding-Mode Robot Control With Exponential Reaching Law[J]. IEEE Transactions on Industrial Electronics, 58(2):600-610(2011).

17. Luo H, Duan X, Deng H. Sliding mode impedance control of an underactuated prosthetic hand[C] IEEE International Conference on Information and Automation. 2014:726-729.

18. Erickson D, Weber M, Sharf I. Contact stiffness and damping estimation for robotic systems [J]. The International Journal of Robotics Research, 22(1): 41-57(2003).

19. Park J, Khatib O. A haptic teleoperation approach based on contact force control [J]. The International Journal of Robotics Research, 25(5-6): 575-591(2006).

20. Wai R J, Su K H. Adaptive enhanced fuzzy sliding-mode control for electrical servo drive [J]. IEEE Transactions on Industrial Electronics, 53(2):569-580(2006). 